\title{
Insights into the regulation of human CNV-miRNAs from the view of their target genes
}

\author{
Xudong Wu, Dinglin Zhang and Guohui Li
}

\begin{abstract}
Background: microRNAs (miRNAs) represent a class of small (typically 22 nucleotides in length) non-coding RNAs that can degrade their target mRNAs or block their translation. Recent research showed that copy number alterations of miRNAs and their target genes are highly prevalent in cancers; however, the evolutionary and biological functions of naturally existing copy number variable miRNAs (CNV-miRNAs) among individuals have not been studied extensively throughout the genome.

Results: In this study, we comprehensively analyzed the properties of genes regulated by CNV-miRNAs, and found that CNV-miRNAs tend to target a higher average number of genes and prefer to synergistically regulate the same genes; further, the targets of CNV-miRNAs tend to have higher variability of expression within and between populations. Finally, we found the targets of CNV-miRNAs are more likely to be differentially expressed among tissues and developmental stages, and participate in a wide range of cellular responses.

Conclusions: Our analyses of CNV-miRNAs provide new insights into the impact of copy number variations on miRNA-mediated post-transcriptional networks. The deeper interpretation of patterns of gene expression variation and the functional characterization of CNV-miRNAs will help to broaden the current understanding of the molecular basis of human phenotypic diversity.
\end{abstract}

Keywords: Copy number variation, miRNA, Expression variation, HapMap ethnic population

\section{Background}

miRNAs are a class of small non-coding RNAs, which act through binding in a sequence-specific manner to the $3^{\prime}$ UTR of target genes [1]. Each miRNA can potentially regulate many transcripts and at least one-third of human genes are estimated to be miRNA targets. miRNAs participate in posttranscriptional gene regulation by repressing the expression of their target genes through inhibition of translation or cleavage of mRNAs [2-6]. miRNAs also contribute to genetic buffering of the gene expression variation, and play an important role in maintaining the identity of mature tissues through a feed-forward loop regulatory architecture $[7,8]$, such as the relationship between miR-9a and $E(s p l)$ in Drosophila $[9,10]$ and the regulation of $E 2 F 1$ by miR-17 in human [11].

A primary goal in medical and evolutionary genomics is to understand the genetic mechanisms of natural

\footnotetext{
*Correspondence: ghli@dicp.ac.cn

Laboratory of Molecular Modeling and Design, State key Laboratory of Molecular Reaction Dynamics, Dalian Institute of Chemical Physics, Chinese Academy of Sciences, 457 Zhongshan Rd., Dalian 116023, PR China
}

variation in gene expression [12-16]. The structure of the human genome is highly variable and the copy number variations $(\mathrm{CNVs})$ refer to alterations of genomic segments of more than 1,000 nucleotides that are present at significant frequencies within a population [17-19]. Many studies showed that CNVs can expand dosage variation of the associated genes, leading to the under-representation of dosage-sensitive protein-coding functional units such as transcription factors and members of protein complexes $[20,21]$. CNVs can be discovered by cytogenetic techniques, such as fluorescent in situ hybridization, comparative genomic hybridization, array comparative genomic hybridization, and nextgeneration sequencing [22-24]. In humans, more than 30,000 genomic regions with segmental duplications have been recognized by systematic comparative genomic hybridizations on the DNA of healthy human subjects; however, the CNVs of other animals were far less studied (see http://projects.tcag.ca/variation). For example, only about 2,000 CNVs have been identified in 
Pan troglodytes [25] and about 4,000 CNVs in inbred Mus musculus [26,27].

Recent studies revealed a high frequency in copy number abnormality of miRNA processing genes, such as Dicer1 and Argonature2, in breast and ovarian cancers $[28,29]$. Although copy number alterations of miRNAs and their regulatory genes were frequently investigated in oncogenesis [28-30], the evolutionary and functional impact of CNV-miRNAs on the human genome has not been studied extensively. Based on the human genomic structure variations, Marcinkowska et al. recently detected about 30\% miRNAs located in the human CNV-regions, indicating that non-coding RNAs also have potential functional variants [31].

In this study, we comprehensively analyzed the properties of genes regulated by CNV-miRNAs and explored the potential involvement of CNV-miRNAs in the expression variability of their targets within and between populations. Our analysis revealed significant functional differences between the targets of CNV-miRNAs and the targets of non-CNV-miRNAs. The involvement of CNV-miRNAs in a wide range of cellular responses provided us with valuable information of the impact of CNVs on the post-transcriptional network.

\section{Results}

\section{Characterization of the regulation of CNV-miRNAs from} the view of their target genes

We first compiled the genes regulated by CNV-miRNAs using the targets from TargetScan5.1 [32], which predicts miRNA targets based on sequence complementarities, sequence context information and binding energy. Because of its high confidence, TargetScan 5.1 has been widely used in a variety of "omics" studies (see Methods) [32-34]. From among the miRNA-Target associations that were obtained, the representative miRNA for each family with the lowest total context score was presented, but all other miRNAs from the same family were considered to target the same gene at the same target sites [34]. To study the non-redundant miRNA binding sites directly, we replaced the miRNAs by their miRNAfamily ID. Finally, 63,428 regulatory relationships were constructed comprising 541 miRNA-families and 9,174 targets (see Additional file 1).

According to the study by Marcinkowska et al. [31], a total of 209 miRNAs were found to locate in the human CNV-regions. These miRNAs belong to 172 families (see Additional file 2); the remaining 369 miRNA-families had no members in the CNV-regions. In the following analysis, these two types were referred to as CNVmiRNAs and non-CNV-miRNAs, respectively.

We investigated target genes of the non-CNVmiRNAs and CNV-miRNAs and classified them into three groups (see Additional file 3). The first group contains a total of 1,134 target genes that are regulated exclusively by $\mathrm{CNV}$-miRNAs, 823 of the genes are regulated by one CNV-miRNA, 211 by two CNV-miRNAs, 67 by three CNV-miRNAs, 22 by four CNV-miRNAs, and 11 by $\geq 5 \mathrm{CNV}$-miRNAs. The second group contains a total of 5,710 target genes that are regulated by non-CNVmiRNAs and at least one CNV-miRNA. The third group consists of 2,330 target genes that are regulated exclusively by non-CNV-miRNAs, 1,408 of the genes are regulated by one non-CNV-miRNA, 515 by two non-CNV-miRNAs, 207 by three non-CNV-miRNAs, 95 by four non-CNVmiRNAs and 105 by $\geq 5$ non-CNV-miRNAs.

To explore the target-recognition preference of CNVmiRNAs and non-CNV-miRNAs, we devised a sampling method to investigate whether the observed number of target genes for each regulatory type could be expected from random sampling. The simulation analysis involved two steps: (a) 172 miRNAs were selected randomly from the 541 miRNAs, and assumed to be pseudo-CNVmiRNAs; (b) in the miRNA-target regulatory network (see Additional file 1), the edges connecting genes and pseudo-CNV-miRNAs, and the edges connecting genes and pseudo-non-CNV-miRNAs were marked, respectively; the number of target genes $(k)$ was recorded for each type. The steps (a) and (b) were repeated 1,000 times, and resulted in normal distributions of target genes for each type of miRNA regulation. The Z-scores and their transformed p-values (calculated by NORMDIST function in Microsoft Excel) were then used to assess the statistical significance of whether the observed number deviated significantly from random expectation. The simulations provide clues to the regulatory patterns of CNV-miRNAs. As shown in Table 1 , the number of genes regulated exclusively by one CNVmiRNA (823 genes were regulated by 137 CNV-miRNAs, approximately 6 target genes per CNV-miRNA) was significantly higher than the number expected from random simulations ( $p \sim 0.05)$. In contrast, the number of genes regulated exclusively by one non-CNV-miRNA $(1,408$ genes were regulated by 280 non-CNV-miRNAs, approximately 5 target genes per non-CNV-miRNA) was significantly lower than the number expected from random simulations ( $\mathrm{p} \sim 0.05)$. Thus CNV-miRNAs tend to target a higher average number of genes compared with nonCNV-miRNAs. Besides, two and more CNV-miRNAs tend to synergistically regulate the same genes; that is, these genes are preferentially targeted by a combination of CNV-miRNAs in which directional selection may be involved in increasing the frequency of CNV-miRNAs in the human genome [35-37]. Obviously, the copy number variation of miRNAs is not independent of copy number variation of the other miRNAs if their binding sites are co-located in the same untranslated regions (UTRs) and regulate the same genes. As shown in Figure 1A for this 
Table 1 Simulation analysis to explore the target-recognition preference of CNV-miRNAs and non-CNV-miRNAs

\begin{tabular}{|c|c|c|c|c|c|}
\hline & $\begin{array}{l}\text { The numberof regulatory } \\
\text { miRNAs }\end{array}$ & $\begin{array}{l}\text { Mean of } 1,000 \\
\text { simulations }\end{array}$ & $\begin{array}{l}\text { Std. dev of } 1,000 \\
\text { simulations }\end{array}$ & $\begin{array}{l}\text { Observed } \\
\text { number }\end{array}$ & $p$-values \\
\hline \multirow{5}{*}{$\begin{array}{l}\text { Genes regulated exclusively } \\
\text { by CNV miRNAs }\end{array}$} & 1 CNV-miRNA & 716.479 & 67.633 & 823 & 0.0576 \\
\hline & 2 CNV-miRNAs & 134.428 & 22.810 & 211 & 0.000392 \\
\hline & 3 CNV-miRNAs & 28.597 & 8.115 & 67 & 0.000001 \\
\hline & 4 CNV-miRNAs & 7.119 & 3.421 & 22 & 0.000006 \\
\hline & $\geq 5$ CNV-miRNAs & 2.809 & 1.846 & 11 & 0.0000004 \\
\hline \multirow{5}{*}{$\begin{array}{l}\text { Genes regulated exclusively } \\
\text { by non-CNV miRNAs }\end{array}$} & 1 non-CNV-miRNA & 1514.503 & 67.633 & 1,408 & 0.0576 \\
\hline & 2 non-CNV-miRNAs & 609.760 & 45.420 & 515 & 0.0184 \\
\hline & 3 non-CNV-miRNAs & 277.837 & 31.374 & 207 & 0.0119 \\
\hline & 4 non-CNV-miRNAs & 145.793 & 22.981 & 95 & 0.0135 \\
\hline & $\geq 5$ non-CNV-miRNAs & 185.483 & 42.717 & 105 & 0.0297 \\
\hline
\end{tabular}

The $\mathrm{p}$-values were obtained from the Z-score, which was calculated as (observed number - mean number of 1000 simulations) / standard deviation of 1000 simulations.

type of co-regulation, miRNA- $\alpha<->$ miRNA- $\beta$, the copy number alteration of miRNA- $\alpha$ could influence copy number alteration of miRNA- $\beta$, or vice versa. Theoretically, it is required that dosage of miRNA- $\alpha$ and miRNA- $\beta$ should be balanced in synergistically regulating the same genes, which may promote the simultaneous retention of concurrent CNV-miRNAs and finally increase reciprocally the number of genes regulated by CNV-miRNAs. To verify this speculation, we analyzed 211 target genes that were regulated exclusively by two CNV-miRNAs, this dataset contained 422 interactions among 211 genes and 113 CNV-miRNAs (see Additional file 3). If CNV-miRNAs were retained or occurred independently, the number of target genes should follow a normal distribution of $\mathrm{N}$ $(134,22)$ (see Table 1 and Figure 1B). Therefore, the number of genes affected by non-independent CNV-miRNAs can be estimated as $211-\mathrm{N}(134,22)=\mathrm{N}(77,22)$ (see Figure 1C). To investigate how many of the CNV-miRNAs were caused by the dosage-balance in co-regulation of the same genes, we (a) removed the information of CNVmiRNAs and then drew a number $(m)$ from a normal distribution $\mathrm{N}(77,22)$, (b) randomly assigned $m$ genes to the miRNA-target regulatory network (see Additional file 1), miRNAs which targeted the selected genes were marked, and their number $(f)$ was recorded. The two steps, (a) and (b), were repeated 1,000 times. $f$ followed a normal distribution as $\mathrm{N}(74,14)$ and was then divided by 2 to give $\mathrm{N}(37,7)$. Thus, the miRNA-target recognition retained about $37 \mathrm{CNV}$ - miRNAs with the standard error of 7 (see Figure 1D); at least one-third (calculated by 37/113) of the CNV-miRNAs were attributable to the requirement of dosage-balance for synergistic regulation.

\section{Target genes of CNV-miRNAs tend to be differentially} expressed among individuals within a population Intuitively, CNVs of miRNA genes can dramatically change their dosage, and this would then affect the expression levels of the target genes in the corresponding individuals $[5,15]$. Recently, a series of genome-wide gene expression profiles have been measured in four HapMap ethnic populations, CEU (U.S. residents with Northern and Western European ancestry), YRI (Yoruba people of Ibadan, Nigeria), $C H B$ (Chinese Han in Beijing) and JPT (Japanese from Tokyo). We calculated the coefficient of variation (CV) for each protein-coding gene across individuals in the four populations to quantify the within-population expression variability of each of the genes (see Methods). Briefly, the CV is the ratio of the standard deviation of gene's expression to its mean intensity, which is considered to be an unbiased and comprehensive metric to measure the regulation diversity at the expression level among individuals [38] (see Additional file 4).

As shown in Figure 2A for the YRI population, the mean CV was 0.0251 for target genes regulated exclusively by non-CNV-miRNAs and increased to 0.0258 for target genes regulated by both CNV-miRNAs and nonCNV-miRNAs ( $\mathrm{p}=0.0110$, Mann-Whitney $U$, two-tail test), the mean $\mathrm{CV}$ was further increased to 0.0274 for target genes regulated exclusively by CNV-miRNAs $(\mathrm{p}=0.0072$, Mann-Whitney $U$, two-tail test). Using the CVs calculated in CEU (Figure 2B), CHB (Figure 2C) and JPT (Figure 2D) populations, we obtained similar results.

The associated sequence variants, such as causative bi-allelic SNPs, could also lead to the different expression variability [12-14,39], we explored whether the minor allele frequencies (MAFs) of SNPs in the target genes of the CNV-miRNAs were significantly different from target genes of non-CNV-miRNAs. The $5^{\prime} \mathrm{UTR}$ and $3^{\prime}$ UTR sequences of human Ensembl genes were downloaded using BioMart [40], and then the HapMap Phase III SNPs (retrieved from http://hgdownload.cse.ucsc. edu/goldenPath/hg19/database/) [41] were mapped onto the sequences (see Methods and Additional file 5). As 


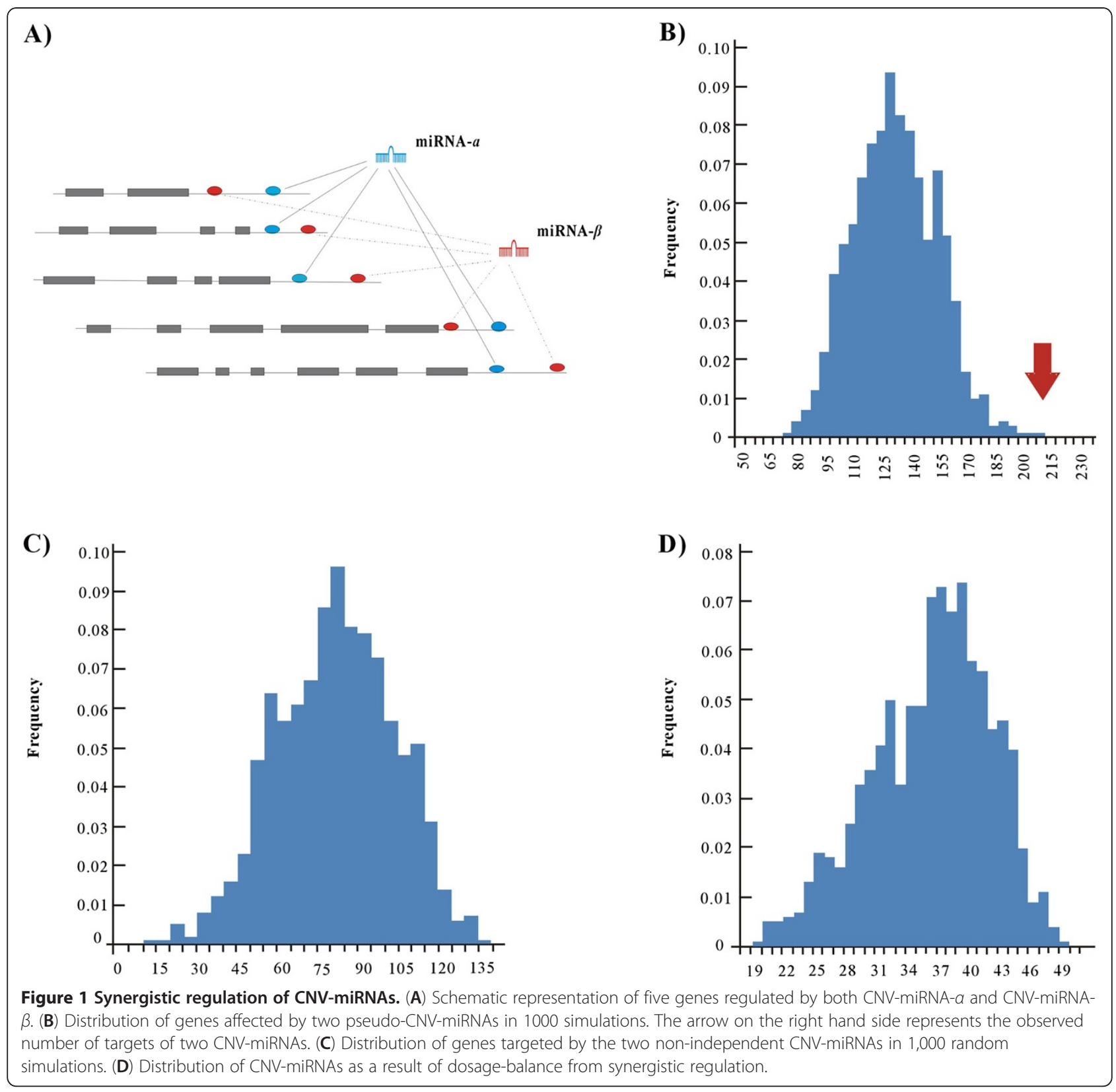

shown in Figure 3, genes regulated exclusively by either non-CNV-miRNAs or CNV-miRNAs have similar proportions of genes that have SNPs in $5^{\prime}$ UTRs and 3'UTRs; furthermore, the SNPs in the $5^{\prime} U T R s$ and 3'UTRs have similar MAFs in each of four HapMap populations ( $\mathrm{p}$-values range from 0.13 to 0.97 , two-tailed t-test). Because genome-wide association and regression analyses have mainly used the MAFs to infer statistical correlations of SNPs with a trait; similar MAFs often indicate that the corresponding SNPs have similar probability to be detected. Therefore, the cis-elements of $5^{\prime} \mathrm{UTR}$ and $3^{\prime} \mathrm{UTR}$ may contain less information than trans-elements in explaining gene expression variations, it is possible that the regulation of some CNV-miRNAs adds a more diversifying control and promotes the differential expression of their target genes among individuals.

Target genes of CNV-miRNAs are more likely to be differentially expressed between populations

A good study has demonstrated that the withinpopulation expression variability of genes can influence the propensity of their differential expression levels between populations [42]. Here, some CNV-miRNAs may live in different populations; thus, the genes targeted by these CNV-miRNAs are likely to be differentially 


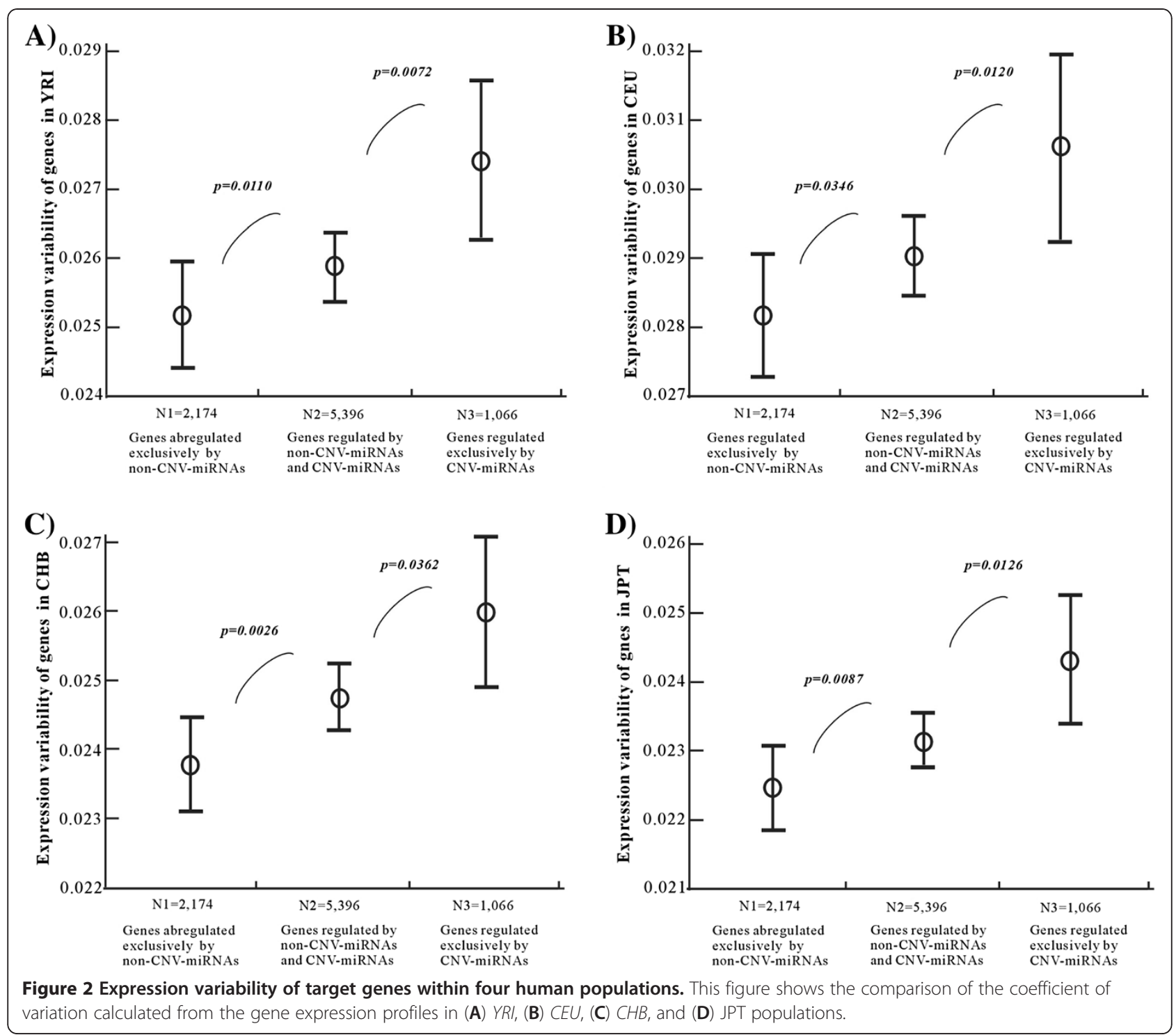

expressed among individuals within a population and also between different populations.

To verify this prediction, we identified the genes that were differentially expressed between any two of the four populations. Taking the CEU and YRI populations as example, we first (a) regress average gene expression intensity, Myri, in YRI and Mceu, in CEU reciprocally; (b) using Myri as the explanatory variable, a liner model was derived by minimizing the square errors between the observed Myri and the predicted values ( ${ }^{\wedge} \mathrm{M} y r i$ ); (c) the residues, $\mathrm{r}=\mathrm{M} y r i$ - ${ }^{\wedge} \mathrm{M} y r i$, were transformed by a quartile normalization and studentized to $\hat{r}$, the outliers were detected according to their ${ }^{\wedge} \mathrm{r}$ away from the calculated 95\% confidence intervals of the t-distribution (see details in $l m$ and rstudent functions of stats $\mathrm{R}$ package http://www.r-project.org/); (d) using $\mathrm{Mceu}$, as the explanatory variable, the two steps (b) and (c) were repeated. As shown in Figure 4A, the mean expression intensities of the genes in the CEU and YRI populations were compared; the red dots in the plot of Myri and Mceu represent genes showing CEU- and YRI-specific variation of expression intensity.

Using the method described above, we identified genes that were differentially expressed in at least one of the four ethnic populations (see Additional file 6). As shown in Figure 4B, a similar number of genes were differentially expressed among six population pairs selected from the four ethnic populations. We then investigated whether genes targeted by CNV-miRNAs were overrepresented in these differentially expressed genes. As shown in Figure 4C, the proportion of differentially expressed genes was $15.7 \%$ for targets regulated exclusively by non-CNV-miRNAs, $17.4 \%$ for targets regulated by both CNV-miRNAs and non-CNV-miRNAs ( $\mathrm{p}=0.060$, 

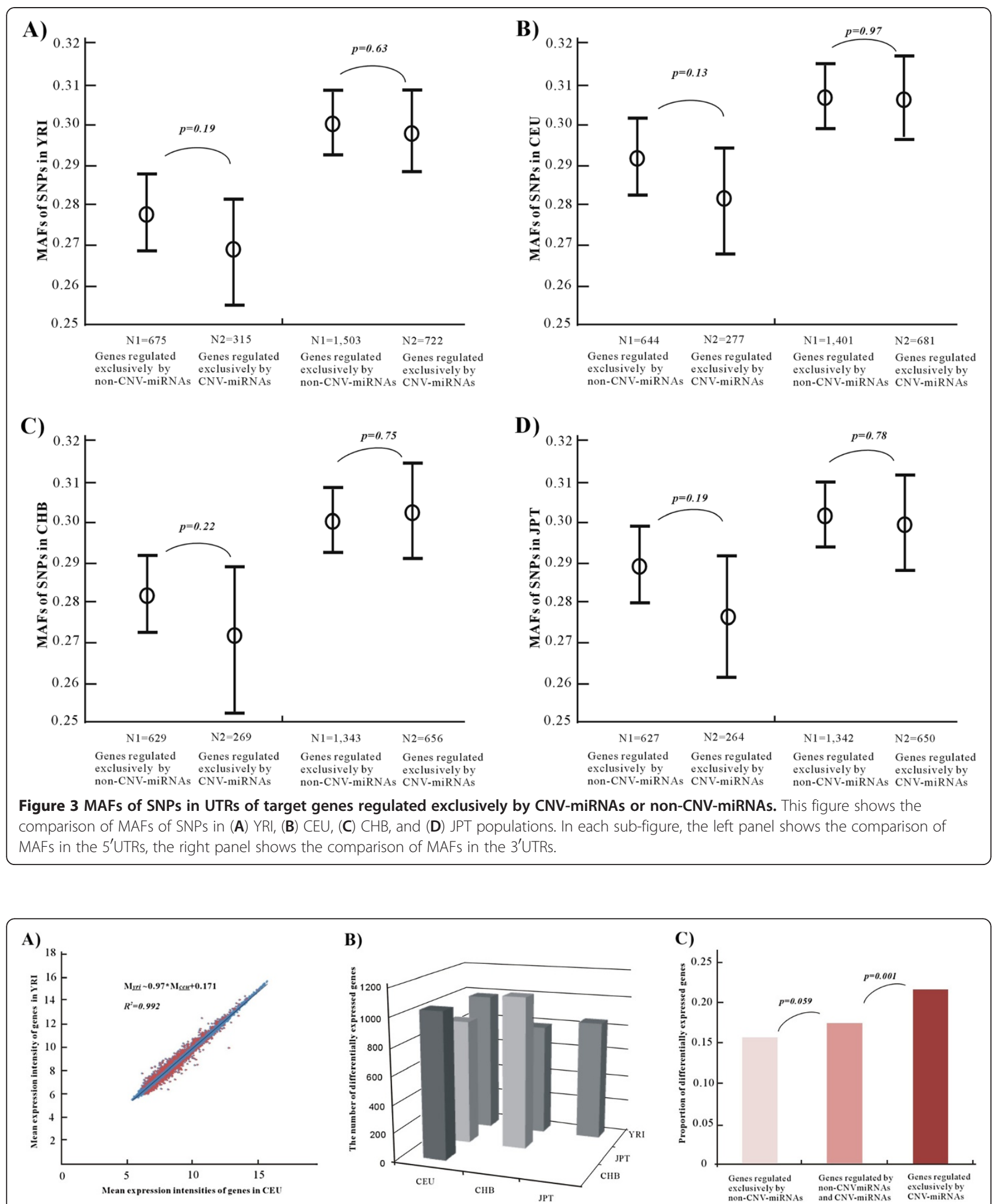

Figure 4 Expression variability of target genes between four human populations. (A) The plot of expression intensities of the genes in the YRI and CEU population, the red dots represent the genes that show CEU- and YRI-specific significantly differential expression intensities.

(B) Number distribution of the differentially expressed genes in six population pairs selected from four ethnic populations. (C) Comparison of proportion of genes differentially expressed in the genes that are regulated exclusively by either non-CNV-miRNAs, non-CNV-miRNAs and CNVmiRNAs, or CNV-miRNAs, respectively. 
Chi-square, two-tail test), the proportion increased further to $21.7 \%$ for targets regulated exclusively by CNV-miRNAs ( $\mathrm{p}=0.001$, Chi-square, two-tail test).

\section{Target genes of CNV-miRNAs tend to be differentially} expressed across tissues and developmental stages

For miRNAs that are specifically expressed in a particular tissue or at a particular developmental stage, the copy number duplication or deletion of miRNAs may lead to either weaker or stronger expression of their target genes in the corresponding tissue and developmental stage. For each human gene, we obtained its Differential Expression Ratio (DER) from the FitSNPs [43]. This DER value was a measure of the frequency of differential expression of the gene in multiple microarray studies across thousands of samples (see Methods). Because the $D E R$ is derived from all available human microarray datasets deposited in NCBI's GEO database (http://www. ncbi.nlm.nih.gov/geo/), it provides a comprehensive metric to measure the regulation diversity of genes at the expression level [44]. As shown in Figure 5, the mean DER was 0.506 for 9,784 genes that are not regulated by miRNAs, 0.514 for 2,249 target genes regulated exclusively by non-CNV-miRNAs ( $\mathrm{p}=1.81 \mathrm{E}-7$, MannWhitney $U$, two-tail test), and increased further to 0.535 for 6,730 target genes of CNV-miRNAs ( $\mathrm{p}=2.36 \mathrm{E}-36$, Mann-Whitney $U$, two-tail test), which include 5,626 targets regulated by non-CNV-miRNAs and CNV-miRNAs, and 1,104 targets regulated exclusively by CNV-miRNAs (see Additional file 7). Therefore, CNV-miRNAs indeed add a more diversifying and complex regulation control to their targets and contribute to an increased likelihood of differential expression among different tissues, cell types, developmental and disease stages.

\section{Functional differences between target genes regulated exclusively by CNV-miRNAs and target genes regulated exclusively by non-CNV-miRNAs}

The Gene Ontology annotation system [45] contained 190,525 associations among 14,117 human genes and 412 GO terms. This data was downloaded and intersected with the 9,174 miRNA target genes that were identified using TargetScan5.1. We obtained GO terms for 6,952 miRNA targets and sought to determine whether the genes that were regulated exclusively by CNV-miRNAs encode proteins that have specific molecular functions or that are involved in particular biological processes (see Methods). As shown in Figure $6 \mathrm{~B}$ and $6 \mathrm{D}$, targets regulated exclusively by non-CNV-miRNAs were significantly enriched for fundamental biological processes such as maintenance of chromatin, organelle and biogenesis, chromosome segregation, extracellular transport and nucleic acid metabolic process. These processes are known to be essential and dosage-sensitive and their radical fluctuation usually reduces an organism's fitness. In contrast, targets regulated exclusively by CNV-miRNAs are enriched for processes responsible for stimulus response, immune response, amino acid

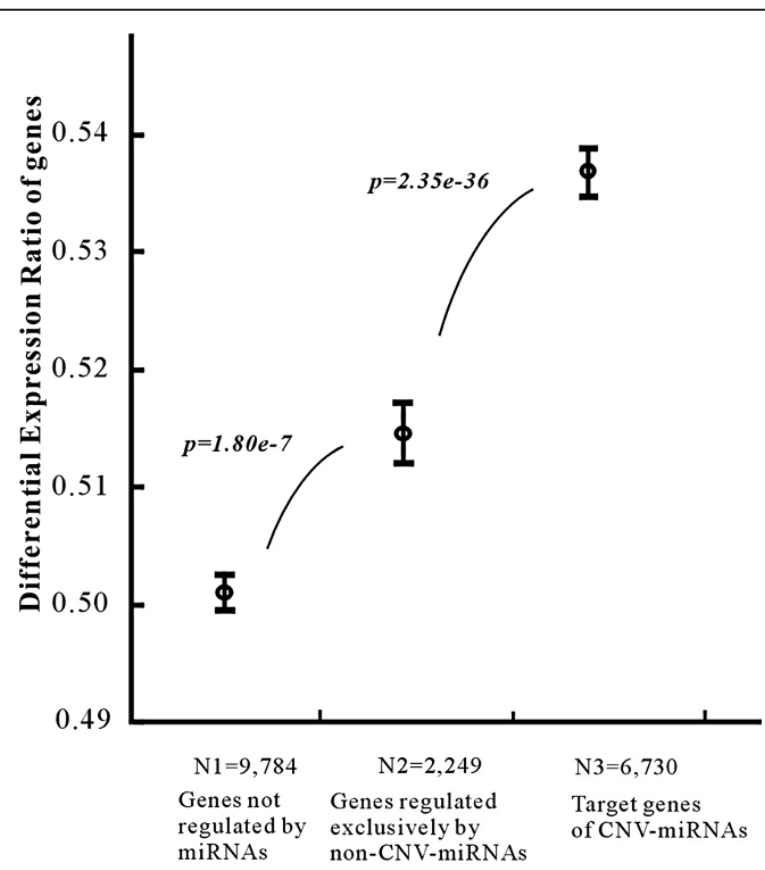

Figure $\mathbf{5}$ Comparison of the differential expression ratios of human genes. Expression variation was measured across 4,877 subset-versus-subset comparisons. 


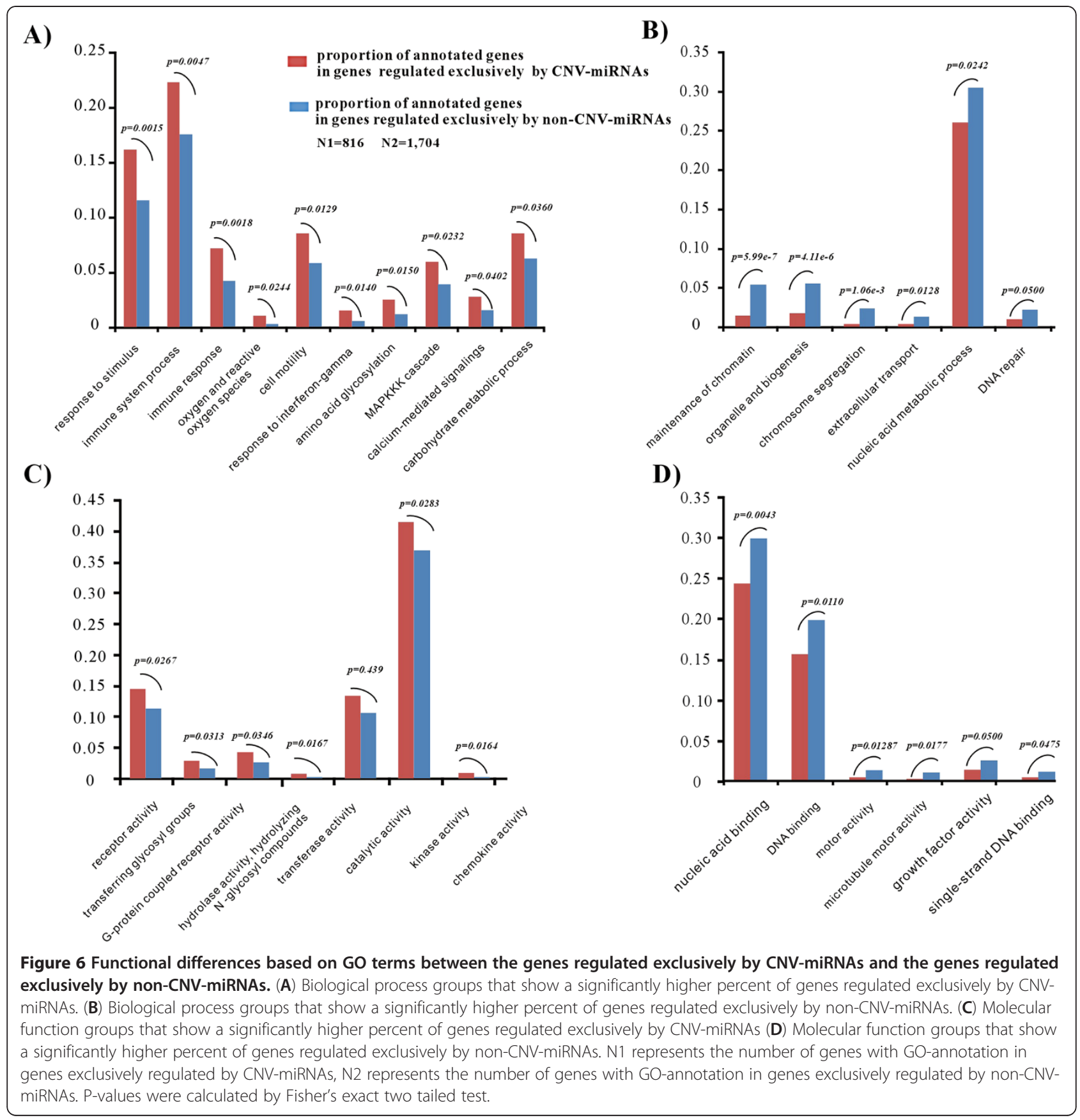

glycosylation and the MAPKKK cascade (Figure 6A and $6 \mathrm{C})$. These processes were environment-oriented and transduce a large variety of external signals, leading to a wide range of cellular responses such as growth, differentiation, inflammation and apoptosis. The flexible regulation for these processes is required and generally provides positive selectiveness to an organism's survival.

\section{Discussion}

It is interesting to know whether or not the orthologs of human CNV-miRNAs were also located in CNV- regions of other animals. We compiled the available CNVs of Pan troglodytes [25] and Mus musculus $[26,27]$, and then intersected the location of their miRNAs with the coordinates of the CNVs. The results showed that only 21 and eight miRNA-families have members located in CNV-regions in Pan troglodytes and inbred Mus musculus, respectively (see Additional file 8). Hence, the human genome contained the highest proportion of CNV-miRNAs, making it the best model to detect the mechanisms and function of CNV-miRNAs. 
Animal genomes have the characteristics of dynamics and plasticity, giving them the ability to adapt to changing environmental conditions. Mobile and evolving elements such as telomeres, transposons, and copy number variants have been studied in investigations into the potential effect of environment on genomes. For example, Haasl and Payseur designed a mathematical model to study microsatellite variations, such as the expected distribution of repeat sizes, and the expected squared difference in repeat size among samples; their simulations revealed that microsatellites, especially triplet repeats, provided adaptation facilitators for beneficial evolution of genomes [46]. miRNAs are relatively newly discovered genomic elements, but their post-transcriptional regulation is present early on in metazoan evolution [47]. The number of miRNAs in a genome correlates with the morphological complexity of the animal, indicating that they play roles in evolutionary changes of body structure [48]. It is now widely accepted that an increase in the complexity of gene regulatory mechanisms, at both the genomic and transcriptomic level, drives the appearance of more complex organisms. Two distinct mechanisms of increasing complexity of gene expression, namely, the co-evolution between CNVs and miRNAs, have been recently recognized and studied. Marcinkowska et al. compared the fractions of miRNA loci and the fraction of genome covered by CNVs, and reported that the CNV purification effect was insignificant [31]. Felekkis et al. demonstrated that the number of distinct miRNA types and the average number of miRNA binding sites in genes in $\mathrm{CNV}$ regions were significantly higher than genes in non-CNV regions [37]. In this study, we proposed the miRNA-target recognition may play important roles in escape from purification of the CNV-miRNAs that target the same genes. Further analysis revealed that "targeting by CNV-miRNAs" seems to be favored and that the target genes participate in a wide-range of cellular responses to environmental factors. For target genes regulated by one miRNA, CNV-miRNAs tend to target a higher average number of genes than non-CNV-miRNAs. From an evolutionary viewpoint, if the CNV-miRNAs were deleterious and only remained in the genome because they were difficult to remove, then we might expect them to have a tendency to target, on average, a lesser number of genes than non-CNV-miRNAs; furthermore, if the CNV-miRNAs were neutral and their retention attributed to random genetic drift, the CNVmiRNAs and non-CNV-miRNAs should target a similar average number of genes. Therefore, some CNVmiRNAs seems to be beneficial to the organism and "targeting by CNV-miRNAs" may provide positive selective pressure to their target genes.

From a biological view, four paradigms could be used to explain the co-evolutionary relationship between
CNVs and miRNAs. In the first paradigm, a simple repression motif is involved where miRNA reduces the expression of its target $(\mathrm{T})$, and the increased dosage due to $\mathrm{CNV}$-duplication of the target $(\mathrm{T})$ is balanced by the corresponding CNV-duplication of miRNA (Figure 7A). In the second paradigm, a miRNA and its target $(\mathrm{T}) \mathrm{mu}-$ tually buffer each other's expression from perturbation in a negative feedback loop, the increased dosage due to CNV-duplication of the target $(\mathrm{T})$ is buffered by the expression variation of the miRNA [49] (Figure 7B). In the third paradigm, the CNV-duplication of some miRNAs can compensate for the CNV-deletion of other miRNAs in balancing the dosage variation of their common target (T) (Figure 7C). In the final paradigm, the common target $(\mathrm{T})$ of two miRNAs is up-regulated in the cellular response to environmental factors, the intrinsic dosagesensitivity of the target (T) makes the CNV-duplication of both the miRNAs favorable (Figure 7D). Obviously, CNVs and miRNAs must have co-evolved complementarily in a tradeoff between maintaining the balance of the dosage-sensitive genes and the increasing diversity of dosage-non-sensitive genes [50]. With genomic plasticity being controlled, CNV-miRNAs provide the possibility of increasing regulatory complexity and the evolvability of genomes.

Our analyses revealed pervasive impacts of $\mathrm{CNV}$ on the miRNA-mediated post-transcription regulatory network. Previous studies demonstrated that miRNAs preferentially regulated the hubs of protein interaction [51] and metabolic networks [52]. We here propose that the $\mathrm{CNV}$ of miRNAs may fluctuate the dosage balance of signal transduction pathways, metabolic flux or protein complexes [53,54], leading eventually to individuals of the same population or different populations having different susceptibility to diseases [55]. Although it is difficult to identify these CNV-miRNAs without a comprehensive investigation of health risks among human populations, recent experimental studies have discovered $\mathrm{CNV}$-causing dysregulation of miRNAs that confirmed their roles in disease occurrence. In one study, nextgeneration sequencing technology was used to explore $\mathrm{CNV}$ as a potential mechanism of miRNA mis-expression, the affected miRNA loci were consistently found to be either lost or gained, and their candidate mRNA targets were coordinately dysregulated; the authors demonstrated the structure variation of the miRNA loci clearly characterized the pre-invasive stage of breast cancer [56]. In another study, genetic networks were inferred from miRNA expression in normal and cancer tissues, and cancer networks built from disjointed sub-networks were found to accompany miRNA copy number alterations, such as the amplification of the hsa-miR-17/92 family, the deletion of the hsa-miR-143/145 cluster, and the physical alteration of the hsa-miR-204/30 at the 


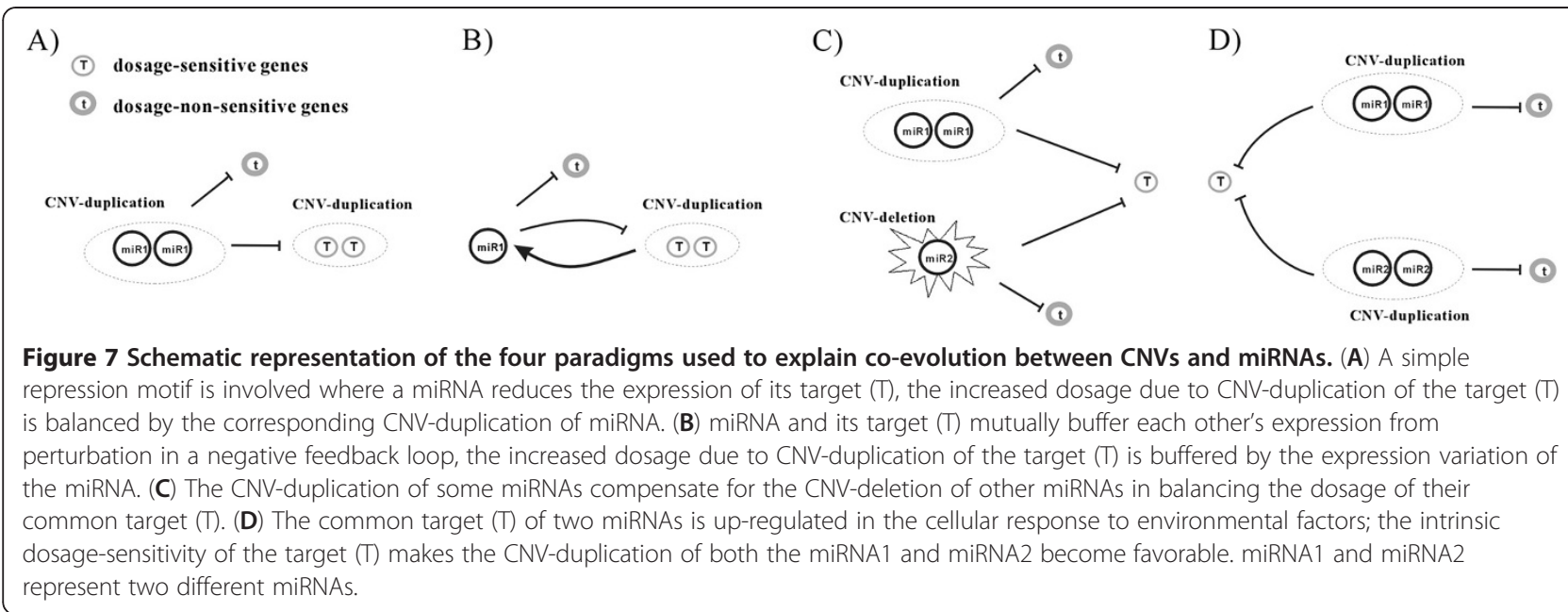

DNA copy number level [57]. The results of these studies clearly demonstrate the feasibility of using the dysregulation of CNV-miRNAs as biological markers for disease screening; indicating that $\mathrm{CNV}$-miRNAs and their targets should be given more attention in studies of human health.

\section{Conclusions}

To the best of our knowledge, this is the first genomewide integrative analysis among human CNVs, miRNAs, their targets and expression variations. Our results will pave the way for future studies for the functional characterization of CNV-miRNAs. This study reveals more clear roles of CNV-miRNAs and is valuable for studying the impact of CNVs on human health.

\section{Methods}

\section{Compilation of human miRNA target genes}

The miRNAs and their predicted targets were taken from TargetScan (http://www.targetscan.org version 5.1) $[32,33]$. Targets with a total context score of -0.3 or lower were ignored, where the score quantitatively measure the overall target efficacy [58]. A total of 9,174 targets with at least one conserved 7-mer or 8-mer were selected as reliable miRNA targets [59] (see Additional file 1).

\section{Analysis of human gene expression data}

The microarray-based gene expression profiles were derived from lymphoblastic cell lines of 270 HapMap individuals (http://www.sanger.ac.uk/humgen/genevar, GSE6536), including 90 samples of YRI (Yoruba people of Ibadan, Nigeria), 90 samples of CEU (U.S. residents with northern and western European ancestry), 45 samples of CHB (Chinese Han in Beijing) and 45 samples of JPT (Japanese from Tokyo) [60,61]. The annotation table was retrieved from http:/www.ncbi.nlm.nih.gov/projects/ geo/query/acc.cgi?acc=GPL2507. The RefSeq identifiers were transformed to Ensembl Gene ID through BioMart [40]. Finally, the expression profiles of 16,686 human genes (including 8,636 miRNA targets) across four HapMap populations were complied.

The following formulas were adopted to calculate the coefficient of variation (CV) of gene $i$ in each ethnic population.

The mean intensity $M_{i}$ calculated by $M_{i}=\frac{\sum_{j=1}^{n} S_{i j}}{n}$
The standard deviation $\sigma_{i}$ calculated by $\sigma_{i}=\sqrt{\frac{\left(S_{i j}-M_{i}\right)^{2}}{n-1}}$,

The coefficient of variation $C V_{i}$ calculated by $C V_{i}=\frac{\sigma_{i}}{M_{i}}$

Where $j=1$ to $n, n$ represents the number of samples in a population, $S_{i j}$ represents the expression signal of gene $i$ in sample $j$. Greater CV implies higher expression variability of a gene across individuals within the corresponding population (see Additional file 4).

\section{Calculation of MAFs of SNPs in UTRs of human genes}

Minor allele frequency (MAF) refers to the frequency at which the less common allele occurs in a given population. SNPs with a minor allele frequency of $5 \%$ or greater were targeted by the HapMap project and have been widely employed in Genome Wide Association Studies for complex traits (GWAS) [62,63].

For a SNP $A / a$, the minor allele frequency was calculated by the following formula

$$
M A F=\frac{\min \left(2 N_{A A}+N_{A a}, 2 N_{a a}+N_{A a}\right)}{\left(2 N_{A A}+2 N_{a a}+N_{A a}\right)}
$$

Where $\mathrm{N}_{a a}$ represents the count of individuals who are homozygous for allele1, $\mathrm{N}_{A a}$ represents the count of individuals who are heterozygous, $\mathrm{N}_{a a}$ represents the count of individuals who are homozygous for allele2. 


\section{Compilation of DERs of human genes}

The differential expression ratios (DER) of human genes were obtained from the study by Chen et al. (FitSNPs, http://fitsnps.stanford.edu/download.php) [43]. Briefly, the authors downloaded 476 human GEO datasets from the NCBI Gene Expression Omnibus and categorized each GEO dataset into 24 types of comparisons, such as disease state, cell type, metabolism and so on. A total of 4,877 subset-versus-subset comparisons were performed to identify differentially expressed genes with a cutoff of $q$ value $\leq 0.05$ by $S A M$ package [44]. For each human gene, the count of GEO datasets in which it was differentially expressed was divided by the count of its measured GEO.

The gene symbols and EntrezGene IDs were transformed to their Ensembl gene IDs using the BioMart program [40].The Ensembl genes with available DERs were then intersected with the genes that were used for TargetScan5.1 prediction. Finally, the DER values of 9,784 genes that are not regulated by miRNAs and 8,979 target genes of miRNAs were obtained.

\section{Functional analysis of human genes based on gene ontology}

The Gene Ontology (GO) has developed three structured controlled vocabularies to describe gene products in terms of their associated biological processes, cellular components and molecular functions [45]. The human gene association file was downloaded from http://www. geneontology.org/gene-associations/. For each GO term, the proportion of annotated genes was compared between the genes regulated exclusively by CNV-miRNAs and the genes regulated exclusively by non-CNV-miRNAs. The p-value was estimated by Fisher's exact two-tailed test, and a cutoff of $\mathrm{p} \leq 0.05$ was used to identify the overrepresented or under-represented GO terms among the genes that are regulated exclusively by CNV-miRNAs.

\section{Computational environment}

The project was started and completed in Dalian Institute of chemical Physics. Computations were performed on a Linux cluster with 50 nodes (Intel 5130, 2.0 GHz CPU, 4G memory, Laboratory of Molecular Modeling and Design, Dalian Institute of Chemical Physics, Chinese Academy of Sciences). Perl (http://perl.org) and R (http://www.r-project.org/ ) scripts were used for analysis, and can be obtained on request.

\section{Additional files}

Additional file 1: The 63,428 regulatory relationships among 541 miRNA families and 9,174 target genes.

Additional file 2: The 172 human CNV-miRNA-families and their encoding members.
Additional file 3: List of targets genes with three regulatory patterns of miRNAs. The numbers in parenthesis represent the total number of regulatory miRNAs and the number of CNV-miRNAs, respectively.

Additional file 4: Coefficient of variation (CV) of human proteincoding genes in four HapMap ethnic populations.

Additional file 5: Minor allele frequencies (MAFs) of 5'UTR- and $3^{\prime}$ UTR-SNPs in four HapMap ethnic populations.

Additional file 6: List of 2,624 differentially expressed genes among six population pairs comparisons selected from four HapMap ethnic populations.

Additional file 7: The differential expression ratios (DERs) of 1,8763 human genes that were used for TargetScan5.1 prediction.

Additional file 8: The 21 and eight CNV-miRNA-families of Pan troglodytes and Mus musculus, respectively. As there are no miRNA targets from the TargetScan prediction for Pan troglodyte, the miRNAfamily IDs were represented by Rfam identifiers (http://rfam.sanger.ac.uk/).

\section{Abbreviations}

CNV: Copy number variation; CNV-miRNA: miRNA that is located in copy number variation regions; non-CNV-miRNA: miRNA that is not located in copy number variation regions; CEU: U.S. residents with northern and western European ancestry; YRI: Yoruba people of Ibadan, Nigeria; CHB: Chinese Han in Beijing; JPT: Japanese from Tokyo; CV: The coefficient of variation ratio; MAF: Minor allele frequency; GO: Gene Ontology.

\section{Competing interests}

The authors declare that they have no competing interests.

\section{Authors' contributions}

XW and GL conceived and designed the study, XW performed the experiments, XW, DZ and GL analyzed the data, XW, DZ and GL wrote the paper. All authors read and approved the final manuscript.

\section{Acknowledgments}

This work was supported by funding from "Hundred Talents Program" of Chinese Academy of Sciences and State key Laboratory of Molecular Reaction Dynamics, Dalian Institute of Chemical Physics, Chinese Academy of Sciences.

Received: 22 April 2012 Accepted: 7 December 2012

Published: 18 December 2012

\section{References}

1. Bartel DP: MicroRNAs: genomics, biogenesis, mechanism and function. Cell 2004, 116:281-297.

2. He L, Hannon GJ: MicroRNAs: small RNAs with a big role in gene regulation. Nat Rev Genet 2004, 5:522-531.

3. Rosero S, Bravo-Egana V, Jiang Z, Khuri S, Tsinoremas N, Klein D, Sabates E, Correa-Medina M, Ricordi C, Domínguez-Bendala J, Diez J, Pastori RL: MicroRNA signature of the human developing pancreas. BMC Genomics 2010, 11:509.

4. Ding XC, Grosshans H: Repression of C. elegans microRNA targets at the initiation level of translation requires GW182 proteins. EMBO J 2009, 28:213-222.

5. Lim LP, Lau NC, Garrett-Engele P, Grimson A, Schelter JM, Castle J, Bartel DP, Linsley PS, Johnson JM: Microarray analysis shows that some microRNAs downregulate large numbers of target mRNAs. Nature 2005, 433:769-773.

6. Vivek J, Mark L, David DF M, Yang YH: Identification of microRNA-mRNA modules using microarray data. BMC Genomics 2011, 12:138.

7. Yu Z, Jian Z, Shen SH, Purisima E, Wang E: Global analysis of microRNA target gene expression reveals that miRNA targets are lower expressed in mature mouse and drosophila tissues than in the embryos. Nucleic Acids Res 2007, 35:152-164.

8. Hornstein E, Shomron N: Canalization of development by microRNAs. Nat Genet 2006, 38:S20-S24.

9. Li Y, Wang F, Lee JA, Gao FB: MicroRNA-9a ensures the precise specification of sensory organ precursors in Drosophila. Genes Dev 2006 20:2793-2805.

10. Cohen SM, Brennecke J, Stark A: Denoising feedback loops by thresholding - a new role for microRNAs. Genes Dev 2006, 20:2769-2772. 
11. O'Donnell KA, Wentzel EA, Zeller Kl, Dang CV, Mendell JT: c-Myc-regulated microRNAs modulate E2F1 expression. Nature 2005, 435:839-843.

12. Morley M, Molony CM, Weber TM, Devlin JL, Ewens KG, Spielman RS, Cheung VG: Genetic analysis of genome-wide variation in human gene expression. Nature 2004, 430:743-747

13. Cheung VG, Spielman RS, Ewens KG, Weber TM, Morley M, Burdick JT: Mapping determinants of human gene expression by regional and genome-wide association. Nature 2005, 437:1365-1369.

14. GuhaThakurta D, Xie T, Anand M, Edwards SW, Li G, Wang SS, Schadt EE: Cis-regulatory variations: a study of SNPs around genes showing cis-linkage in segregating mouse populations. BMC Genomics 2006, 7:235.

15. Henrichsen CN, Chaignat E, Reymond A: Copy number variants, diseases and gene expression. Hum Mol Genet 2009, 18(R1):R1-R8.

16. Pickrell JK, Marioni JC, Pai AA, Degner JF, Engelhardt BE, Nkadori E, Veyrieras JB, Stephens M, Gilad Y, Pritchard JK: Understanding mechanisms underlying human gene expression variation with RNA sequencing. Nature 2010, 464:768-772.

17. Wong KK, deLeeuw RJ, Dosanjh NS, Kimm LR, Cheng Z, Horsman DE, MacAulay C, Ng RT, Brown CJ, Eichler EE, Lam WL: A comprehensive analysis of common copy-number variations in the human genome. Am J Hum Genet 2007, 80:91-104.

18. Bonaglia MC, Giorda R, Beri S, De Agostini C, Novara F, Fichera M, Grillo L, Galesi O, Vetro A, Ciccone R, Bonati MT, Giglio S, Guerrini R, Osimani S, Marelli S, Zucca C, Grasso R, Borgatti R, Mani E, Motta C, Molteni M, Romano C, Greco D, Reitano S, Baroncini A, Lapi E, Cecconi A, Arrigo G, Patricelli MG, Pantaleoni C, D'Arrigo S, Riva D, Sciacca F, Dalla Bernardina B, Zoccante L, Darra F, Termine C, Maserati E, Bigoni S, Priolo E, Bottani A, Gimelli S, Bena F, Brusco A, di Gregorio E, Bagnasco I, Giussani U, Nitsch L, Politi P, Martinez-Frias ML, Martínez-Fernández ML, Martínez Guardia N, Bremer A, Anderlid BM, Zuffardi O: Molecular mechanisms generating and stabilizing terminal 22q13 deletions in 44 subjects with Phelan/ McDermid Syndrome. PLoS Genet 2011, 7:e1002173.

19. Conrad DF, Pinto D, Redon R, Feuk L, Gokcumen O, Zhang Y, Aerts J Andrews TD, Barnes C, Campbell P, Fitzgerald T, Hu M, Ihm CH, Kristiansson K, Macarthur DG, Macdonald JR, Onyiah I, Pang AW, Robson S, Stirrups K, Valsesia A, Walter K, Wei J, Wellcome Trust Case Control Consortium, TylerSmith C, Carter NP, Lee C, Scherer SW, Hurles ME: Origins and functional impact of copy number variation in the human genome. Nature 2010, 464:704-712.

20. Wang RT, Sangtae A, Park CC, Khan AH, Kenneth L, Smith DJ: Effects of genome-wide copy number variation on expression in mammalian cells. BMC Genomics 2011, 12:562.

21. Woodwark C, Bateman A: The characterization of three types of genes that overlie copy number variable regions. PLoS One 2011, 6(5):e14814

22. Korbel JO, Urban AE, Affourtit JP, Godwin B, Grubert F, Simons JF, Kim PM, Palejev D, Carriero NJ, Du L, Taillon BE, Chen Z, Tanzer A, Saunders AC, Chi J, Yang F, Carter NP, Hurles ME, Weissman SM, Harkins TT, Gerstein MB, Egholm M, Snyder M: Paired-end mapping reveals extensive structural variation in the human genome. Science 2007, 318:420-426.

23. Sudmant PH, Kitzman JO, Antonacci F, Alkan C, Malig M, Tsalenko A Sampas N, Bruhn L, Shendure J, 1000 Genomes Project, Eichler EE: Diversity of human copy number variation and multicopy genes. Science 2010, 330:641-646.

24. Mills RE, Walter K, Stewart C, Handsaker RE, Chen K, Alkan C, Abyzov A, Yoon SC, Ye K, Cheetham RK, Chinwalla A, Conrad DF, Fu Y, Grubert F, Hajirasouliha I, Hormozdiari F, lakoucheva LM, lqbal Z, Kang S, Kidd JM, Konkel MK, Korn J, Khurana E, Kural D, Lam HY, Leng J, Li R, Li Y, Lin CY, Luo R, Mu XJ, Nemesh J, Peckham HE, Rausch T, Scally A, Shi X, Stromberg MP, Stütz AM, Urban AE, Walker JA, Wu J, Zhang Y, Zhang ZD, Batzer MA, Ding L, Marth GT, McVean G, Sebat J, Snyder M, Wang J, Ye K, Eichler EE, Gerstein MB, Hurles ME, Lee C, McCarroll SA, Korbel JO, 1000 Genomes Project: Mapping copy number variation by population-scale genome sequencing. Nature 2011, 470:59-65.

25. Perry GH, Yang F, Marques-Bonet T, Murphy C, Fitzgerald T, Lee AS, Hyland C, Stone AC, Hurles ME, Tyler-Smith C, Eichler EE, Carter NP, Lee C, Redon R: Copy number variation and evolution in humans and chimpanzees. Genome Res 2008, 18:1698-1710.

26. Cutler G, Marshall LA, Chin N, Baribault H, Kassner PD: Significant gene content variation characterizes the genomes of inbred mouse strains. Genome Res 2007 , 17:1743-1754.
27. Agam A, Yalcin B, Bhomra A, Cubin M, Webber C, Holmes C, Flint J, Mott R: Elusive copy number variation in the mouse genome. PLOS One 2010, 5(9):e12839.

28. Zhang L, Huang J, Yang N, Greshock J, Megraw MS, Giannakakis A, Liang S, Naylor TL, Barchetti A, Ward MR, Yao G, Medina A, O'brien-Jenkins A Katsaros D, Hatzigeorgiou A, Gimotty PA, Weber BL, Coukos G: microRNAs exhibit high frequency genomic alterations in human cancer. Proc Natl Acad Sci USA 2006, 103:9136-9141.

29. Lionetti M, Agnelli L, Mosca L, Fabris S, Andronache A, Todoerti K, Ronchetti $D$, Deliliers GL, Neri A: Integrative high-resolution microarray analysis of human myeloma cell lines reveals deregulated miRNA expression associated with allelic imbalances and gene expression profiles. Genes Chromosomes Cancer 2009, 48:521-531.

30. Maire G, Martin JW, Yoshimoto M, Chilton-MacNeill S, Zielenska M, Squire JA: Analysis of miRNA-gene expression-genomic profiles reveals complex mechanisms of microRNA deregulation in osteosarcoma. Cancer Genet 2011, 204:138-146.

31. Marcinkowska M, Szymanski M, Krzyzosiak WJ, Kozlowski P: Copy number variation of microRNA genes in the human genome. BMC Genomics 2011, $12: 183$.

32. Lewis BP, Burge CB, Bartel DP: Conserved seed pairing, often flanked by adenosines, indicates that thousands of human genes are microRNA targets. Cell 2005, 120:15-20.

33. Chen K, Rajewsky N: Natural selection on human microRNA binding sites inferred from SNP data. Nat Genet 2006, 38:1452-1456.

34. Grimson A, Farh KK, Johnston WK, Garrett-Engele P, Lim LP, Bartel DP: MicroRNA targeting specificity in mammals: determinants beyond seed pairing. Mol Cell 2007, 27:91-105.

35. Fay JC, Wyckoff GJ, Wu Cl: Positive and negative selection on the human genome. Genetics 2001, 158:1227-1234.

36. Nielsen R, Hellmann I, Hubisz M, Bustamante C, Clark AG: Recent and ongoing selection in the human genome. Nat Rev Genet 2007, 8:857-868.

37. Felekkis K, Voskarides K, Dweep H, Sticht C, Gretz N, Deltas C: Increased number of microRNA target sites in genes encoded in CNV regions, Evidence for an evolutionary genomic interaction. Mol Biol Evol 2011, 28:2421-2424

38. Kaern M, Elston TC, Blake WJ, Collins JJ: Stochasticity in gene expression: from theories to phenotypes. Nat Rev Gene 2005, 6:451-464.

39. Hartl D: A Primer of Population Genetics. 3rd edition. Sunderland, MA, USA: Sinauer Associates, Inc.; 2000

40. Smedley D, Haider S, Ballester B, Holland R, London D, Thorisson G, Kasprzyk A: BioMart-biological queries made easy. BMC Genomics 2009, 10:22

41. The International HapMap Consortium: Integrating common and rare genetic variation in diverse human populations. Nature 2010, 467:52-58.

42. Li J, Liu Y, Kim T, Min R, Zhang Z: Gene expression variability within and between human populations and implications toward disease susceptibility. PLoS Comput Biol 2010, 6(8):e1000910.

43. Chen R, Morgan AA, Dudley J, Deshpande T, Li L, Kodama K, Chiang AP, Butte AJ: FitSNPs: highly differentially expressed genes are more likely to have variants associated with disease. Genome Biol 2008, 9:R170.

44. Chen R, Li L, Butte AJ: AlLUN: reannotating gene expression data automatically. Nat Methods 2007, 4:879.

45. Day-Richter J, Harris MA, Haendel M, Gene Ontology OBO-Edit Working Group, Lewis S: OBO-Edit-an ontology editor for biologists. Bioinformatics 2007, 23:2198-2200.

46. Haasl RJ, Payseur BA: The number of alleles at a microsatellite defines the allele frequency spectrum and facilitates fast accurate estimation of theta. Mol Biol Evol 2010, 12:2702-2715.

47. Sempere LF, Cole CN, McPeek MA, Peterson KJ: The phylogenetic distribution of metazoan microRNAs: insights into evolutionary complexity and constraint. J Exp Zool B Mol Dev Evol 2006, 306:575-588.

48. Heimberg AM, Sempere LF, Moy VN, Donoghue PC, Peterson KJ: MicroRNAs and the advent of vertebrate morphological complexity. Proc Natl Acad Sci USA 2008, 105(8):2946-2950.

49. Wu Cl, Shen $Y$, Tang T: Evolution under canalization and the dual roles of microRNAs-A hypothesis. Genome Res 2009, 19(5):734-743.

50. Zhou J, Lemos B, Dopman EB, Hartl DL: Copy-number variation: the balance between gene dosage and expression in drosophila melanogaster. Genome Biol Evol 2011, 3:1014-1024.

51. Liang $H$, Li WH: MicroRNA regulation of human protein-protein interaction network. RNA 2007, 13(9):1402-1408. 
52. Tibiche $C$, Wang E: MicroRNA regulatory patterns on the human metabolic network. The Open Systems Biology Journal 2008, 1:1-8.

53. Veitia RA: Gene dosage balance in cellular pathways: implications for dominance and gene duplicability. Genetics 2004, 168:569-574.

54. Veitia RA, Bottani S, Birchler JA: Cellular reactions to gene dosage imbalance: genomic, transcriptomic and proteomic effects. Trends Genet 2008, 24:390-397.

55. Knight JC: Human Genetic Diversity: Functional Consequences for Health and Disease. 1st edition. Oxford, UK: Oxford University Press; 2009.

56. Bethany Noelle Hannafon: An integrated analysis of the coordinated dysregulation of microRNAs and their targets in pre-invasive breast cancer. In PhD thesis.: Boston University; 2010.

57. Volinia S, Galasso M, Costinean S, Tagliavini L, Gamberoni G, Drusco A, Marchesini J, Mascellani N, Sana ME, Abu Jarour R, Desponts C, Teitell M, Baffa R, Aqeilan R, lorio MV, Taccioli C, Garzon R, Di Leva G, Fabbri M, Catozzi M, Previati M, Ambs S, Palumbo T, Garofalo M, Veronese A, Bottoni A, Gasparini P, Harris CC, Visone R, Pekarsky Y, de la Chapelle A, Bloomston M, Dillhoff M, Rassenti LZ, Kipps TJ, Huebner K, Pichiorri F, Lenze D, Cairo S, Buendia MA, Pineau P, Dejean A, Zanesi N, Rossi S, Calin GA, Liu CG, Palatini J, Negrini M, Vecchione A, Rosenberg A, Croce CM: Reprogramming of miRNA networks in cancer and leukemia. Genome Res 2010, 20(5):589-599.

58. Baek D, Villen J, Shin C, Camargo FD, Gygi SP, Bartel DP: The impact of microRNAs on protein output. Nature 2008, 455:64-71.

59. Wu X, Song Y: Preferential regulation of miRNA targets by environmental chemicals in the human genome. BMC Genomics 2011, 12:244.

60. Stranger BE, Forrest MS, Dunning M, Ingle CE, Beazley C, Thorne N, Redon $R$, Bird CP, de Grassi A, Lee C, Tyler-Smith C, Carter N, Scherer SW, Tavaré S, Deloukas P, Hurles ME, Dermitzakis ET: Relative impact of nucleotide and copy number variation on gene expression phenotypes. Science 2007, 315:848-853.

61. Stranger $B E$, Nica AC, Forrest MS, Dimas A, Bird CP, Beazley $C$, Ingle $C E$, Dunning M, Flicek P, Koller D, Montgomery S, Tavaré S, Deloukas $P$, Dermitzakis ET: Population genomics of human gene expression. Nat Genet 2007, 39:1217-1224.

62. Serre D, Gurd S, Ge B, Sladek R, Sinnett D, Harmsen E, Bibikova M, Chudin E, Barker DL, Dickinson T, Fan JB, Hudson TJ: Differential allelic expression in the human genome: a robust approach to identify genetic and epigenetic cis-acting mechanisms regulating gene expression. PLoS Genet 2008, 4(2):e1000006

63. Spencer CC, Su Z, Donnelly P, Marchini J: Designing genome-wide association studies: sample size, power, imputation, and the choice of genotyping chip. PLoS Gene 2009, 5(5):e1000477.

doi:10.1186/1471-2164-13-707

Cite this article as: Wu et al:: Insights into the regulation of human CNVmiRNAs from the view of their target genes. BMC Genomics 2012 13:707.

\section{Submit your next manuscript to BioMed Central and take full advantage of:}

- Convenient online submission

- Thorough peer review

- No space constraints or color figure charges

- Immediate publication on acceptance

- Inclusion in PubMed, CAS, Scopus and Google Scholar

- Research which is freely available for redistribution

Submit your manuscript at www.biomedcentral.com/submit
() Biomed Central 